\title{
The Lipid Composition and Permeability to the Triazole Antifungal Antibiotic ICI 153066 of Serum-grown Mycelial Cultures of Candida albicans
}

\author{
By CHRISTOPHER A. HITCHCOCK,${ }^{1}+$ KEITH J. BARRETT-BEE ${ }^{2}$ AND \\ NICHOLAS J. RUSSELL ${ }^{1 *}$ \\ ${ }^{1}$ Department of Biochemistry, University of Wales College of Cardiff, PO Box 903 , \\ Cardiff CF1 1ST, UK \\ ${ }^{2}$ Pharmaceuticals Division, ICI plc, Alderley Park, Macclesfield SK10 4TG, Cheshire, UK
}

(Received 5 January 1989; revised 10 April 1989; accepted 12 April 1989)

\begin{abstract}
The total lipid content of Candida albicans (serotype A; NCPF 3153) exponential-phase mycelial cultures grown in tissue-culture medium 199 (containing $10 \%, \mathrm{v} / \mathrm{v}$, foetal calf serum) was $29.8 \pm 8 \mathrm{mg}$ (g dry weight $)^{-1}$ (mean $\pm \mathrm{SD}$ ). The weight ratios of phospholipid to neutral lipid and phospholipid to non-esterified sterol were $2.6 \pm 0.4$ and $24.9 \pm 0.5$, respectively. The major phospholipid was phosphatidylcholine with smaller amounts of phosphatidylethanolamine, phosphatidylinositol, phosphatidylserine, phosphatidylglycerol and diphosphatidylglycerol; the most abundant fatty acids were palmitic, palmitoleic, oleic and linoleic acids. The major neutral lipids comprised esterified sterol, triacylglycerol and non-esterified fatty acid with a smaller amount of non-esterified sterol. The fatty acid compositions of the three fatty-acid-containing neutral lipids were distinct from each other and the phospholipids. Comparison with previous data on yeast cultures of $C$. albicans $\mathrm{A}$ grown in glucose broth shows that mycelial cultures have a larger lipid content, lower phospholipid to neutral lipid ratio and higher phospholipid to none.--ified sterol ratio. We now show that mycelial cultures were more permeable to a $\left[{ }^{14} \mathrm{C}\right]$ triazole antifungal antibiotic compared with exponentially growing yeast cultures of several azole-sensitive strains. Taken together these data are consistent with there being a relationship between the phospholipid/non-esterified sterol ratio of a culture and its ability to accumulate a triazole.
\end{abstract}

\section{INTRODUCTION}

Candida albicans is a widespread opportunistic fungal pathogen responsible for superficial and systemic candidoses (Odds, 1988). The fungus is dimorphic, growing as either a budding yeast or filamentous mycelium, and both forms are invariably present in lesions. The mycelial form appears not to be essential for virulence in vivo, but the weight of evidence suggests that it is important in the pathogenesis of candidoses (for a review see Odds, 1988). The morphological transformation is promoted by a large number of factors in vitro (Odds, 1985), but the most important factor in vivo is probably serum.

The azole ( $N$-substituted imidazole and triazole) class of antifungal antibiotics is used in the treatment of both superficial and deep-seated candidoses (Fromtling, 1988). One characteristic of all the azoles is that they inhibit mycelial growth, which is an important mode of action in view of the suggested pathogenicity of this morphological form (Borgers, 1985). The MIC values

† Present address: Department of Microbiology, University of Leeds, Leeds LS2 9JT, UK.

Abbreviations: TCM, tissue culture medium 199 containing $10 \%(\mathrm{v} / \mathrm{v})$ foetal calf serum; YMA, yeast morphology agar; YNB, yeast nitrogen base; MIC, minimum inhibitory concentration. 
of the azoles in vitro vary considerably with the conditions used in the sensitivity tests and this phenomenon is associated especially with the yeast-to-mycelial transformation (Odds, 1980; Borgers, 1985). However, in sensitivity tests containing serum the order of potency among azoles corresponds more closely to their efficacy in vivo (Borgers, 1985). This situation may reflect the presence of the mycelial form in infections.

The azoles probably work by inhibiting ergosterol biosynthesis in C. albicans by binding to $14 \alpha$-sterol demethylase, an important enzyme in the ergosterol biosynthetic pathway (Van den Bossche et al., 1983). Compared with yeast cultures, ergosterol biosynthesis is more sensitive to azoles in mycelial cultures, and this observation has been used to explain the efficacy of azoles in vivo (Van den Bossche et al., 1980).

However, another possible factor in determining the sensitivity of mycelial cells could be their ability to accumulate azole antifungal antibiotics. There is evidence that ketoconazole is taken up by C. albicans via a saturable, active-transport process (Boiron et al., 1987). Although it is not known how the triazole ICI 153066 enters $C$. albicans, we have shown that yeast cultures of different strains (including azole-resistant strains) have quite different permeabilities to this antifungal antibiotic (Hitchcock et al., 1986, 1987a, b). Furthermore, on the basis of a comparison of the membrane lipid composition of the strains, it was suggested that there was a relationship between the phospholipid to non-esterified sterol ratio of a strain and its ability to take up triazole. Shimokawa et al. (1986) suggested that an altered membrane sterol composition of some mutants of $C$. albicans was responsible for their increased sensitivity to a range of drugs.

In this paper, we report the detailed lipid composition and permeability to triazole of mycelial cultures of $C$. albicans (serotype A), grown in tissue-culture medium 199, supplemented with foetal calf serum. This medium was used as a source of serum because it resembles the situation in vivo, with the expectation of changes in fungal membrane lipid composition that could clarify the efficacy of azoles in vivo. Compared with yeast cultures, mycelial cultures grown in tissueculture medium have a larger phospholipid to non-esterified sterol ratio and are more permeable to a triazole antifungal.

\section{METHODS}

Materials. Phospholipid and neutral lipid standards were purchased from Sigma. Solvents and other chemicals were of analytical grade and were purchased from BDH. The radioactive triazole $\left[{ }^{14} \mathrm{C}\right] \mathrm{ICI} 153066$ was synthesized at ICI Pharmaceuticals Division, Alderley Park, Macclesfield, UK.

Organism and culture conditions. Stock cultures of Candida albicans (serotype A; NCPF 3153) were maintained on slopes of yeast morphology agar (YMA, Difco) as described by Hitchcock et al. (1986). Late-exponential-phase mycelial cultures were grown in 1 litre of tissue culture medium 199 (Gibco) supplemented with $10 \%(\mathrm{v} / \mathrm{v})$ foetal calf serum (Sigma), in 2 litre Erlenmeyer flasks at $37^{\circ} \mathrm{C}$ without shaking for $16 \mathrm{~h}$; the medium was inoculated with $3 \times 10^{4}$ yeasts washed from an agar slope with sterile distilled water. After incubation for $3 \mathrm{~h}, 92 \pm 2 \%$ (mean \pm SD) of the inoculum had formed germ-tubes, as measured by haemocytometer counts. Mycelia grew as a thin white mat on the bottom of the flask and were harvested by filtration through filter paper under negative pressure. Filtered mycelia were washed thoroughly with $2 \times 1$ litre of deionized water before transfer to a roundbottom flask for shell-freeze-drying. Freeze-dried mycelia from 1 litre cultures weighed $60 \pm 10 \mathrm{mg}$.

Late-exponential-phase yeast cultures were grown in yeast nitrogen base containing $2 \%(\mathrm{v} / \mathrm{v})$ glucose (YNB) as described by Hitchcock et al. (1986).

Protein and DNA estimations. The protein and DNA contents were measured in homogenates of mycelia broken by three passages through a French pressure cell (American Instrument $\mathrm{Co}$.), at $1.3 \times 10^{5} \mathrm{kPa}$. Protein was estimated by the method of Lowry using a bovine serum albumin standard. DNA was estimated by the method of Leyva \& Kelly (1974) using a calf thymus DNA standard.

Lipid extraction and analysis. Lipids were extracted from freeze-dried cultures by the method of Bligh \& Dyer (1959) after phospholipase activities had been inactivated with hot 2-isopropanol (Hitchcock et al., 1986). Nonlipid contaminants were removed from total lipid extracts with Sephadex G-25 (Wells \& Dittmer, 1963). The purified lipid was fractionated into individual phospholipids and neutral lipids by thin-layer chromatography. Phospholipids were quantified by estimation of their phosphorus and fatty acid content, and acyl lipids by their fatty acid content. Esterified and non-esterified sterols were identified and quantified by GLC and GC-MS. The details of these methods are given in Hitchcock et al. (1986).

Measurement of azole uptake. Freshly harvested mycelia were washed with $0 \cdot 1 \mathrm{M}$-potassium phosphate buffer, pH 7.4, containing $0.1 \%(\mathrm{v} / \mathrm{v})$ Tween 80 and $0.1 \mathrm{M}$-potassium phosphate, $\mathrm{pH} 5.5$. The uptake of 
Table 1. Fatty acid composition of the total phospholipid and the major individual phospholipids in C. albicans mycelial cultures

The total lipid was fractionated into total phospholipid or individual phospholipids by TLC, and fatty acid methyl esters prepared by transmethylation, as described in Methods. Values are means \pm SD of at least two GLC analyses of fatty acids in phospholipids from three separate cultures. PL, phospholipid; PC, phosphatidylcholine; PE, phosphatidylethanolamine; PI, phosphatidylinositol; PS, phosphatidylserine; ND, not detected; UFA, unsaturated fatty acid; DBI, double bond index.

\begin{tabular}{lccccc}
\multicolumn{5}{c}{ Composition $(\%, w / w) \pm$ SD } \\
\cline { 2 - 6 } Fatty acid & Total PL & PC & PE & PI & PS \\
$16: 0$ & $17.8 \pm 0.6$ & $11.4 \pm 0.6$ & $26.8 \pm 0.2$ & $28.7 \pm 0.3$ & $20.1 \pm 0.3$ \\
$16: 1$ & $14.9 \pm 0.1$ & $16.6 \pm 1.3$ & $12.2 \pm 0.6$ & $4.7 \pm 0.7$ & $18.4 \pm 0.5$ \\
$16: 2$ & $0.9 \pm 0.1$ & $2.2 \pm 0.9$ & $1 \cdot 7 \pm 0.5$ & $5.1 \pm 0.3$ & $2.7 \pm 0.7$ \\
$18: 0$ & $7.5 \pm 0.4$ & $13.9 \pm 0.8$ & $14.4 \pm 0.8$ & $13.9 \pm 1.1$ & $3.0 \pm 0.1$ \\
$18: 1$ & $35.6 \pm 1.6$ & $32.1 \pm 1.5$ & $36.0 \pm 0.9$ & $36.9 \pm 1.0$ & $31.5 \pm 0.5$ \\
$18: 2$ & $20.9 \pm 0.2$ & $22.0 \pm 0.2$ & $8.9 \pm 0.2$ & $11.5 \pm 0.6$ & $20.2 \pm 1.8$ \\
$18: 3$ & $2.2 \pm 0.3$ & $3.0 \pm 0.5$ & ND & ND & ND \\
$\%$ UFA & 75.5 & 75.9 & 58.8 & 58.2 & 72.8 \\
$18 / 16$ ratio & 2.0 & 2.35 & 1.46 & 1.62 & 1.33 \\
DBI & 1.04 & 1.06 & 0.69 & 0.75 & 0.96
\end{tabular}

[U-14 C]ICI 153066 (specific radioactivity $19 \cdot 4 \mathrm{Ci} \mathrm{mg}^{-1}, 718 \mathrm{GBq} \mathrm{mg}^{-1}$ ) by mycelial cell suspensions (50-70 mg wet weight $\mathrm{ml}^{-1}$ ) was measured by the method of Hitchcock et al. (1986), except that assays were terminated by filtering, as described by Ryley et al. (1984).

The uptake of radiolabel by yeast cell suspensions $\left(10^{9}\right.$ cells $\left.\mathrm{ml}^{-1}\right)$ was measured using the method described in Hitchcock et al. (1986). Routinely, parallel control experiments with autoclave-killed cells were done to establish the amount of radiolabel binding to cells. That amount was reproducibly $30 \%$ of the radioactivity incorporated.

\section{RESULTS}

\section{Lipid content}

The amount of total lipid extracted from late-exponential mycelial cultures was $29 \cdot 8 \pm 8 \mathrm{mg}$ lipid (g dry weight) $)^{-1}$, or $290 \pm 20 \mu \mathrm{g}$ lipid (mg total cellular protein) ${ }^{-1}$, or $13.6 \pm 1.3 \mathrm{mg}$ (mg total cellular DNA) $)^{-1}$ (means $\pm \mathrm{SD} ; n=3$ ), respectively. The weight ratios of phospholipid to neutral lipid and phospholipid to non-esterified sterol were $2.6 \pm 0.4$ and $24.9 \pm 0.5(n=3)$, respectively.

\section{Polar lipid composition}

Polar lipids comprised $72 \cdot 2 \pm 0 \cdot 5 \%(n=3)$ of the total lipid and consisted entirely of phospholipid. The phospholipid composition was characterized by a large content of phosphatidylcholine, which accounted for $54.9 \pm 1.4 \%$ of the total phospholipid. There were smaller amounts of phosphatidylethanolamine $(12.3 \pm 0.8 \%)$, phosphatidylinositol $(15.0 \pm 0.5 \%)$, phosphatidylserine $(6 \cdot 7 \pm 0.4 \%)$, phosphatidylglycerol $(2 \cdot 8 \pm 0.2 \%)$ and diphosphatidylglycerol $(9 \cdot 5 \pm 0.6 \%$ ), and a trace $(<1 \%)$ of sphingomyelin (values are means $\pm \mathrm{SD}$ of experiments on three separate cultures). The fatty acid compositions of individual phospholipids differed, and data for the major phospholipids and total phospholipid are shown in Table 1 .

The most abundant fatty acids in each phospholipid were 16:0, 16:1, 18:1 and 18:2. Phosphatidylcholine was the most unsaturated phospholipid which was reflected in a high percentage unsaturated fatty acid and double bond index. It was characterized by a high content of $16: 1$ relative to $16: 0$ and was the only phospholipid to contain $18: 3$. Phosphatidylethanolamine and phosphatidylinositol were the most saturated phospholipids due mainly to their relatively large content of $16: 0$, giving them a low percentage unsaturated fatty acid and double bond index. 
Table 2. Fatty acid composition of non-esterified fatty acid and acyl neutral lipids in C. albicans mycelial cultures

Non-esterified fatty acid and acyl neutral lipid were fractionated from total lipid and fatty acids analysed as described in Methods. Values are means \pm SD of at least two GLC analyses of neutral lipids from three separate cultures. NEFA, non-esterified fatty acid; TAG, triacylglycerol; ES, esterified sterol; ND, not detected; UFA, unsaturated fatty acid; DBI, double bond index.

\begin{tabular}{lccc} 
& \multicolumn{3}{c}{ Composition $(\%, w / w) \pm$ SD } \\
\cline { 2 - 4 } Fatty acid & NEFA & \multicolumn{1}{c}{ TAG } & ES \\
$16: 0$ & $27.6 \pm 1.6$ & $18.6 \pm 0.4$ & $14.6 \pm 0.4$ \\
$16: 1$ & $10.5 \pm 2.5$ & $5.7 \pm 0.8$ & $15.5 \pm 0.4$ \\
$16: 2$ & $3.4 \pm 1.4$ & $2.3 \pm 0.6$ & $8.2 \pm 0.6$ \\
$18: 0$ & $12.6 \pm 1.5$ & $29.3 \pm 1.5$ & $14.8 \pm 0.3$ \\
$18: 1$ & $32.3 \pm 0.8$ & $37.2 \pm 0.6$ & $41.5 \pm 0.5$ \\
$18: 2$ & $13.2 \pm 2.3$ & $8.6 \pm 0.4$ & $6.3 \pm 0.4$ \\
$18: 3$ & $2.0 \pm 1.5$ & ND & ND \\
$\%$ UFA & 61.5 & 53.8 & 71.5 \\
$18 / 16$ ratio & 1.45 & 2.82 & 1.63 \\
DBI & 0.82 & 0.65 & 0.86
\end{tabular}

Table 3. Non-esterified and esterified sterol compositions of $C$. albicans mycelial cultures

Sterols were fractionated from the total lipid by TLC and analysed by GLC and GC-MS as described in Methods. Values are the means of two analyses of sterols from two separate cultures (values varied by $<2 \%$ ). NES, non-esterified sterol; ES, esterified sterol.

\begin{tabular}{lcc} 
& \multicolumn{2}{c}{ Composition $(\%, w / w)$} \\
\cline { 2 - 3 } \multicolumn{1}{c}{ Sterol } & NES & ES \\
Ergosterol & 57.0 & $55 \cdot 3$ \\
Ergosta-7-ene & $24 \cdot 5$ & 25.7 \\
Ergosta-5,7-dien-3 $\beta$-ol & 10.0 & 12.7 \\
Ergosta-7,22-dien-3 $\beta$-ol & 4.0 & 3.2 \\
24-Methylene-24,25-dihydrolanosterol & 3.0 & 2.5 \\
Zymosterol & 1.5 & 0.9
\end{tabular}

\section{Neutral lipid composition}

The neutral lipid comprised $27.8 \pm 0.8 \%(n=3)$ of the total lipid and consisted mainly of triacylglycerol $(39.1 \pm 1.0 \%)$, esterified sterol $(27.7 \pm 0.8)$ and non-esterified fatty $(20.4 \pm 0.8 \%)$ acid with smaller amounts of non-esterified sterol $(8.8 \pm 0.4 \%)$ and squalene $(4.2 \pm 1.8 \%)$ (values are means \pm SD of experiments on three separate cultures). The fatty acid compositions of the three acyl neutral lipids were different from each other (Table 2) and the phospholipids (cf. Table 1).

The esterified sterol was the most unsaturated of the acyl lipids ( $71.5 \%$ unsaturated fatty acid) due to the relatively large proportion of $18: 1$. In contrast, the triacylglycerol was the most saturated of the acyl lipids and contained a large proportion of $18: 0$, giving it a relatively high $\mathrm{C} 18 / \mathrm{Cl} 6$ ratio and small percentage unsaturated fatty acid and double bond index.

The sterol compositions of the esterified and non-esterified sterols were similar (Table 3). Both compositions were notable for the low content of ergosterol, which accounted for approximately half of the total sterols, the remainder of which were metabolic precursors of ergosterol.

\section{Triazole uptake}

The results of a typical experiment comparing the uptake of $\left[{ }^{14} \mathrm{C}\right] \mathrm{ICI} 153066$ by yeast and mycelial cultures of $C$. albicans $\mathrm{A}$ are shown in Fig. 1 . The amount of radioactivity incorporated 


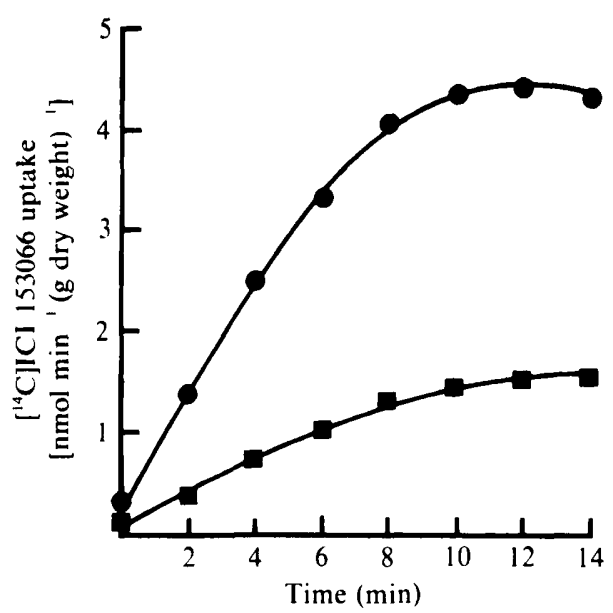

Fig. 1. Uptake of $\left[{ }^{14} \mathrm{C}\right] \mathrm{ICI} 153066$ by exponential-phase mycelial (๑) and yeast $(\boldsymbol{\square})$ cultures of C. albicans. Values for each time point are the mean of triplicate determinations on cells from one culture (values varied by $<10 \%$ ).

by both cultures was linear for $8 \mathrm{~min}$, after which the uptake of triazole plateaued. The rate of uptake of ICI 153066 by mycelial cultures was $0.43 \pm 0.03 \mathrm{nmol} \mathrm{min}^{-1}(\mathrm{~g} \text { dry weight })^{-1}$ compared with $0.15 \pm 0.02 \mathrm{nmol} \mathrm{min}^{-1}$ ( $\mathrm{g}$ dry weight) $^{-1}$ for yeast cultures (values are the means $\pm S D$ of experiments on three separate batches of exponential-phase cultures). The value for yeast cultures corresponds to $2.46 \mathrm{pmol} \mathrm{min}^{-1}\left(10^{9}\right.$ yeasts) ${ }^{-1}$ (calculated on the basis that $10^{9}$ yeasts $=16 \pm 1 \mathrm{mg}$ dry weight) which is in good agreement with $2.52 \mathrm{pmol} \mathrm{min}^{-1}$ $\left(10^{9} \text { yeasts }\right)^{-1}$ given by Hitchcock et al. (1986).

\section{DISCUSSION}

The lipid content of mycelial cultures grown in serum-containing tissue-culture medium is approximately 11 times that of yeast cultures grown in YNB (Hitchcock et al., 1986). This compares closely to the value of 14 times obtained by Davies \& Denning (1972) using mycelial and yeast cultures grown in a similar way to that in the present study. It is apparent that serumgrown mycelial cultures contain more lipid than those grown in serum-free media (Yamaguchi, 1974; Ghannoum et al., 1986), which may reflect the incorporation of serum lipids into fungal cells in addition to de novo synthesis. In addition, the total lipid contains proportionately more neutral lipid relative to polar lipid: the ratio of polar lipid to neutral lipid in mycelial cultures is 2.6 compared with 3.6 in yeast cultures grown in YNB (Hitchcock et al., 1986).

Ghannoum et al. (1986), working with C. albicans ATCC 10231, reported morphology-related differences in phospholipid and neutral lipid compositions between yeast and mycelial forms grown together in mixed culture. However, in the present study with $C$. albicans $A$ it is not known whether the differences in lipid composition between mycelial and yeast (Hitchcock $e t$ al., 1986) cultures are related directly to the morphological change or different media or both. The same argument applies to other published changes in phospholipid (Marriott, 1975; Sundaram et al., 1981; Yano et al., 1982) and neutral lipid (Marriott, 1975; Sundaram et al., 1981) compositions that accompany mycelial growth under a variety of growth conditions. Furthermore, there are no data on mycelial cultures grown in serum-supplemented tissue-culture media for comparison.

Mycelial cultures of $C$. albicans A grown in tissue-culture medium contained the same phospholipids as yeast cultures grown in glucose broth (Hitchcock et al., 1986) but the composition was quite different, due mainly to the large amount of phosphatidylcholine in the mycelial form (54.9 compared with $25.4 \%$, w/w). However, there are similarities in the phospholipid compositions of both morphological forms. For example, phosphatidylcholine and 
phosphatidylinositol were the most unsaturated and saturated (expressed in terms of percentage unsaturated fatty acid) phospholipids, respectively. The large amount of phosphatidylcholine in mycelial cells contributes greatly to the large percentage unsaturated fatty acid value of the total phospholipid. Mycelial phospholipids in other strains are also more unsaturated than those of the yeast form (Yano et al., 1982; Ghannoum et al., 1986), and it has been suggested that the increased fluidity of membranes which this causes might be important for mycelial growth (Yano et al., 1982). The activities of several membrane-bound enzymes depend on fluidity (for a review see Sanderman, 1978), and although it is not known if chitin synthase shows such a dependence, this enzyme is twice as active in the plasma membrane of mycelial cells (Braun \& Calderone, 1978).

In common with the polar lipids, the mycelial neutral lipid composition was distinct from that of the yeast form (Hitchcock et al., 1986). Mycelial cultures contained more esterified sterol and less non-esterified sterol. The significance of the relatively large amount of esterified sterol (27.7 compared with $8.1 \%$, w/w in yeast cultures) is not known: it may be a storage lipid and/or a membrane component (Grover \& Cushley, 1979). In addition, the esterified sterol fatty acyl composition was more unsaturated in mycelial cells $(71.5$ compared with $47.2 \%$, w/w unsaturated fatty acid in yeast cultures).

Although both morphological forms contained the same proportion of triacylglycerol, the fatty acyl compositions were different, the mycelial triacylglycerol being more saturated (53.8 compared with $80.7 \%$ unsaturated fatty acid).

Mycelial lipid contained a significant amount of non-esterified fatty acid, which is thought to be the product of (lyso)phospholipase digestion (Christie, 1982). However, the fatty acid composition was unlike that of any phospholipid (or the total phospholipid), indicating that the enzyme(s) lacked specificity for a particular phospholipid. The same is true for (lyso)phospholipases in yeast cultures of a variety of $C$. albicans strains (Hitchcock et al., 1986, 1987a).

We have shown recently that different strains of $C$. albicans yeasts take up the triazole ICI 153066 at a rate that is proportional to their phospholipid/non-esterified-sterol ratio (Hitchcock et al., 1986). The small amount of non-esterified sterol in mycelial cultures (8.9 compared with $23.8 \%$, w/w in the yeast form), together with the large amount of phospholipid, accounts for the high phospholipid/non-esterified sterol ratio, which is 29.7 in the mycelial form and 15.4 in the yeast form. Moreover, comparison of the phospholipid/ergosterol ratios reveals an even larger difference between the two morphological forms ( 51.3 in the mycelial form and 15 in yeast form), because ergosterol comprises only $57 \%$ of the total non-esterified sterol in mycelial cells. If it is assumed that this ratio for whole cells reflects that of membranes, then together with the more unsaturated phospholipids, mycelial membranes are expected to be considerably more fluid. Such a change in fluidity probably would alter passive diffusion or active transport of the triazole, depending on its mechanism of uptake (Russell, 1989) and, therefore, could explain the increased permeability of mycelial cells to ICI 153066, which may be an important factor in determining the potency of this triazole against the mycelial growth phase (Ryley et al., 1984). The data for mycelial cultures are consistent with there being a relationship between triazole permeability and the ratio of phospholipid/non-esterified sterol. However, it is not known if the ratio determined for total cellular lipid reflects that of the plasma membrane and/or other membranes, and currently we are investigating the mechanism of uptake of triazole in cells from yeast and mycelial cultures. It would be interesting to know if the proposed relationship between lipid composition and uptake of ICI 153066 extended to other triazole antifungal antibiotics.

We should like to thank Mrs Anne Oldfield for performing the GC-MS analysis of sterols. The financial support of the Science and Engineering Research Council is acknowledged.

\section{REFERENCES}

Bligh, E. G. \& DYER, W. J. (1959). A rapid method of total lipid extraction and purification. Canadian Journal of Biochemistry and Physiology 37, 911-917.
BoIron, P., Drouhet, E., DUPONT, B. \& IMProvisi, L. (1987). Entry of ketoconazole into Candida albicans. Antimicrobial Agents and Chemotherapy 31, 244-248. 
Borgers, M. (1985). Antifungal Azole Derivatives. In The Scientific Basis of Antimicrobial Chemotherapy, pp. 133-153. Edited by D. Greenwood \& F. O'Grady. Cambridge: Cambridge University Press.

Braun, P. C. \& Calderone, R. A. (1978). Chitin synthesis in Candida albicans: comparison of yeast and hyphal forms. Journal of Bacteriology 133, 14721477.

Christie, W. W. (1982), Lipid Analysis, 2nd edn. Oxford: Pergamon Press.

DAvies, R. R. \& DenNing, T. J. V. (1972). Growth and form in Candida albicans. Sabouraudia 10, 180-188.

Fromtling, R. A. (1988). Overview of medically important antifungal azole derivatives. Clinical Microbiology Reviews 1, 187-217.

Ghannoum, M. A., Janini, G., Khamis, L. \& RADWAN, S. S. (1986). Dimorphism-associated variations in the lipid composition of Candida albicans. Journal of General Microbiology 132, 2367-2375.

Grover, A. K. \& Cushley, R. J. (1979). Cholesterol ester distribution in lecithin bilayer membranes. Atherosclerosis 32, 87-91.

HitchCock, C. A., Barrett-Bee, K. J. \& Russell, N. J. (1986). The lipid composition of azole-sensitive and azole-resistant strains of Candida albicans. Journal of General Microbiology 132, 2421-2431.

HitchCock, C. A., BarRetT-BeE, K. J. \& Russell, N. J. (1987a). The lipid composition and permeability to azole of an azole- and polyene-resistant mutant of Candida albicans. Journal of Medical and Veterinary Mycology 25, 29-37.

HitchCock, C. A., Russell, N. J. \& Barrett-Bee, K. J. (1987b). Sterols in Candida albicans mutants resistant to polyene or azole antifungals, and of a double mutant C. albicans 6.4. CRC Critical Reviews in Microbiology 15, 111-115.

Leyva, A. \& Kelley, W. N. (1974). Measurement of DNA in cultured human cells. Analytical Biochemistry 62, 173-179.

MARRIOTT, M. S. (1975). Isolation and chemical characterization of plasma membranes from the yeast and mycelial forms of Candida albicans. Journal of General Microbiology 86, 115-132.

ODDs, F. C. (1980). Laboratory evaluation of antifungal agents : a comparative study of five imidazole derivatives of clinical importance. Journal of Anti- microbial Chemotherapy 6, 749-761.

ODDs, F. C. (1985). Morphogenesis in Candida albicans. CRC Critical Reviews in Microbiology 12, 45-93.

ODDs, F. C. (1988). Candida and Candidosis, 2nd edn. London: Ballière Tindall.

RusSELL, N. J. (1989). Functions of lipids: structural roles and membrane functions. In Microbial Lipids, vol. 2, ch. 17. Edited by C. Ratledge \& S. G. Wilkinson. London: Academic Press (in the Press).

Ryley, J. F., Wilson, R. G. \& BARRETt-BeE, K. J. (1984). Azole resistance in Candida albicans. Sabouraudia 22, 53-63.

SANDERMANN, H. (1978). Regulation of membrane enzymes by lipids. Biochimica et biophysica acta 515, 209-237.

Shimokawa, O., Kato, Y. \& Nakayama, H. (1986). Increased drug sensitivity in Candida albicans cells accumulating 14-methylated sterols. Journal of Medical and Veterinary Mycology 24, 481-483.

Sundaram, S., Sullivan, P. A. \& Shepherd, M. G. (1981). Changes in lipid composition during starvation and germ-tube formation in Candida albicans. Experimental Mycology 5, 140-147.

VAN DEN Bossche, H. V., Willemsens, G., CoOls, W., Cornelissen, F., Lauwers, W. F. \& Van Cutsem, J. M. (1980). In vitro and in vivo effects of the antimycotic drug ketoconazole on sterol biosynthesis. Antimicrobial Agents and Chemotherapy 17, 922-928.

VAN DEN Bossche, H. V., Willemsens, G., COOLS, W., Marichal, P. \& LaUwers, W. (1983). Hypothesis on the molecular basis of the antifungal activity of $\mathrm{N}$-substituted imidazoles and triazoles. Biochemical Society Transactions 11, 665-666.

Wells, M. A. \& DitTmer, J. C. (1963). The use of Sephadex for the removal of nonlipid contaminants from lipid extracts. Biochemistry 2, 1259-1263.

YAMAGUCHI, H. (1974). Mycelial development and chemical alteration of Candida albicans from biotin insufficiency. Sabouraudia 12, 320-328.

Yano, K., Yamada, T., Banno, Y., Sekiya, T. \& NozAWA, Y. (1982). Modification of lipid composition in a dimorphic fungus, Candida albicans during the yeast to hypha transformation. Japanese Journal of Medical Mycology 23, 159-165. 